\title{
Nachhaltige dramapädagogische Sprachförderung für Grundschulkinder mit DaZ
}

\author{
Ein durchgängiges Förderkonzept: Vom ausserschulischen \\ Feriencamp zur fachsensiblen Sprachförderung in schulischer \\ Theater-AG
}

Doreen Bryant Sophie Charlotte Rummel

\begin{abstract}
Zusammenfassung
Vielerorts finden in den Schulferien Theatercamps statt, die vor allem sprachschwachen Kindern eine Chance bieten, ihre Sprachkenntnisse spielerisch und handlungsgebunden zu erweitern. Anschlussprojekte, die die Nachhaltigkeit der Fördereffekte sichern und auf diesen aufbauen, gibt es hingegen kaum. Es fehlt an bereichsübergreifenden Konzepten, die im Camp gewonnenen positiven Einstellungen gegenüber Sprache und Schrift in den Schulalltag zu transferieren. Diesem Desiderat begegnend wird in Tübingen derzeit ein durchgängiges Förderkonzept für Grundschulkinder mit Deutsch als Zweitsprache (DaZ) und Sprachförderbedarf erprobt: Im Rahmen von Theater-AGs werden die Kinder, die zur Zeit des Feriencamps die Klasse 2 besuchten, zwei weitere Jahre gefördert, die Kinder der Klasse 3 mindestens ein Jahr. In einem solchen Förderzeitraum ist es möglich, systematisch die Sprachkompetenz aufzubauen und die GrundschülerInnen auf die bildungssprachlichen Anforderungen der Sekundarstufe I vorzubereiten. Während sich die thematisch-inhaltlichen Vorgaben für die Sprachförderung im Camp aus dem gemeinsam zu entwickelnden und aufzuführenden Theaterstück ergeben, werden in den Theater-AGs Inhalte aus dem Schulfach MeNuK (Mensch, Natur, Kultur) dramapädagogisch erarbeitet. Im folgenden Beitrag wird die Entwicklung vom außerschulischen Ferien-Theatercamp hin zum Anschlussprojekt der fachsensiblen Sprachförderung in schulischen Theater-AGs noch einmal im Detail nachvollzogen, wobei exemplarische Fördereinheiten die bereichsübergreifende methodische Kontinuität illustrieren.
\end{abstract}

\section{Einleitung}

Schulen und Lehrkräfte sind zunehmend mit sprachlicher Heterogenität in der Schülerschaft konfrontiert (Arbeitsgruppe Bildungsberichterstattung, 2012). Bereits 2012 gaben 70\% der Lehrkräfte an, SchülerInnen mit Sprachförderbedarf in ihrer Klasse zu haben (Becker-Mrotzek et al. 2012). Im Zuge 
anhaltender Einwanderung aus Euro-Krisenländern und in Folge zunehmender Flüchtlingswellen aus Kriegsregionen hat sich die Situation weiter verschärft. Um sprachbasierte schulische Leistungsnachteile zu verringern, braucht es methodische Konzepte, die fachbezogen an bildungssprachliche Anforderungen heranführen und Sprach- und Fachlernen miteinander verknüpfen (u.a. Benholz et al. 2010; Becker-Mrotzek et al. 2013). Auch unsere praxisbezogene Forschung widmet sich diesem Anliegen.

Im vorliegenden Beitrag soll ein Projekt vorgestellt werden, das gleich in zweierlei Hinsicht Neuland betritt. Zum einen handelt es sich um eine kontinuierliche Maßnahme, die sich an das zweiwöchige Ferien-Theatercamp anschließt, um so die Nachhaltigkeit der Fördereffekte zu sichern und die im Camp gewonnenen positiven Einstellungen gegenüber Sprache und Schrift in den Schulalltag zu transferieren. Dies gelingt mit Theater-AGs, in denen wie im Camp die dramagrammatische Methode (Even 2003; Bryant 2012) Anwendung findet. Unseres Wissens gibt es neben Hamburg und Tübingen bislang keine weiteren Standorte, die entsprechende Nachhaltigkeitskonzepte umsetzen. Die zweite Neuerung ist, dass wir die im Camp erfolgreich erprobte dramagrammatische Methode nun gezielt einsetzen, um Inhalte eines Unterrichtsfaches mit den Kindern zu erarbeiten. Somit wird in den dramagrammatischen Sequenzen nicht nur das sprachliche sondern auch (im Sinne der englischen Tradition von Drama in Education) das fachliche Wissen gefördert. Dem Prinzip der durchgängigen Sprachbildung (u.a. Gogolin et al. 2011) folgend, werden die GrundschülerInnen systematisch auf die sprachlichen Anforderungen der Sekundarstufe I vorbereitet. Dies geschieht über die Inhalte des Faches MeNuK (Mensch, Natur, Kultur). Da im MeNuK-Unterricht die fachlichen, lexikalischen, sprachstrukturellen und textsortenspezifischen Grundlagen für die naturwissenschaftlichen UND für die gesellschaftswissenschaftlichen Fächer der Sekundarstufe gelegt werden (vgl. Benholz \& Rau 2013), bietet es sich in Erwartung einer maximalen Ausstrahlung der Förderimpulse - natürlich an, die Sprachfördermaßnahme an eben dieses Fach zu knüpfen.

Der Beitrag zeigt noch einmal die Chronologie vom außerschulischen FerienTheatercamp hin zum Anschlussprojekt der fachsensiblen Sprachförderung im Rahmen schulischer Theater-AGs. Einerseits wird damit die Projektentwicklung der letzten fünf Jahre nachgezeichnet, andererseits kann so der Interventionspfad nachempfunden werden, den wir den GrundschülerInnen mit Sprachförderbedarf anbieten, um sie sprachlich wie fachlich aufschließen zu lassen und um ihnen so einen erfolgreichen Übergang in die Sekundarstufe zu ermöglichen.

\section{Tübinger Theatercamp}

\subsection{Konzeption des Theatercamps}

Das Tübinger Theatercamp ist einerseits ein Ferienprojekt für Kinder mit Migrationshintergrund und/oder mit Sprachförderbedarf, das in enger Ko- 
operation von der Stadt Tübingen, dem Landestheater und der Universität im Abstand von zwei Jahren durchgeführt wird. Andererseits ist es auch ein pädagogisch und fachwissenschaftlich betreutes Praktikumsangebot des Deutschen Seminars (Bryant 2013). Nach einem intensiven Vorbereitungsseminar betreuen 18 Studierende aus den Studiengängen Lehramt Deutsch und B.A. Deutsch als Zweitsprache - Sprachdiagnostik und Sprachförderung in den Pfingstferien über einen Zeitraum von zwei Wochen 60 bis 66 Kinder der Klassenstufen 2 bis 4 und erarbeiten mit ihnen ein Theaterstück, das am letzten Tag öffentlich aufgeführt wird. Die Kinder sind entsprechend ihres zuvor ermittelten Sprachentwicklungsstandes in sechs relativ homogene Gruppen aufgeteilt und können so optimal in ihrer sprachlichen Entwicklung gefördert werden. Jeweils drei Studierende sind verantwortlich für eine Gruppe von ca. zehn bis zwölf Kindern. Die bereits im Vorfeld überlegte Rahmenhandlung des zu entwickelnden Theaterstücks berücksichtigt die spezifische Camp-Struktur von sechs Gruppen unterschiedlichen Sprachniveaus und beinhaltet dementsprechend sechs sprachlich mehr oder weniger anspruchsvolle Schlüsselszenen mit jeweils zehn bis zwölf zu besetzenden Rollen. So gab es beispielsweise 2013 im Stück „Die glücklichen Fischer“ (Insa Griesing) folgende sechs Gruppen: Fischer, Handwerker, Drachenfischer, Regierung, Forscher, Reporter. Während die Kinder der Fischer-Gruppe kaum Deutsch sprechen konnten, waren die Kinder der Reporter-Gruppe relativ weit fortgeschritten, obgleich man auch ihnen (im Kontrast zu ihren Klassenkameraden) Sprachförderbedarf attestieren musste. Die für die erstgenannte sprachschwächste Gruppe verantwortlichen Studierenden erarbeiteten zusammen mit den Kindern Szenen, die das unbeschwerte, glückliche Lebensgefühl der Fischer vermitteln sollten, vgl. Abbildung 1 und 2 . Hingegen ging es bei der Reporter-Gruppe um die Inszenierung von Interviews, Reportagen und Nachrichten - sprachlich also ein deutlich anspruchsvolleres Unterfangen.

Der Campalltag gliedert sich in Vormittags- und Nachmittagsaktivitäten. Am Vormittag führen die Studierenden in ihrer Gruppe eine szenenbezogene dramagrammatische Sprachförderung durch (siehe 2.2). Am Nachmittag wird die Sprache bei verschiedenen theaterbezogenen Tätigkeiten (Schauspiel, Bewegung, Tanz, Gesang) implizit gefördert. Auch haben die Gruppen nachmittags Gelegenheit, an den Kulissen (u.a. Hütten) zu bauen und an ihren Requisiten und Kostümen zu arbeiten, wobei insbesondere bei den sprachschwachen Kindern auf eine handlungsbegleitende Sprache geachtet wird. Inhaltlich werden Vor- und Nachmittagsaktivitäten zusammengehalten durch das übergeordnete Ziel der Entwicklung und Aufführung eines Theaterstücks. Unterstützt durch szenenbasierte Gruppennamen (Fischer, Forscher, Reporter, ...), eine szenengerechte Gruppenraumausgestaltung (siehe Abbildung 1) sowie durch Gruppenrituale und -kleidung wird zum einen die Gruppenidentität und die Identifikation mit der Szene und den Charakteren gefördert und zum anderen eine Brücke zwischen Vormittags- und Nachmittagsaktivitäten geschlagen (Bryant 2012: 33f). Die inhaltliche Verknüpfung von expliziter und impliziter Sprachförderung, von Vormittags- und Nachmittagsaktivitäten ist eines der 


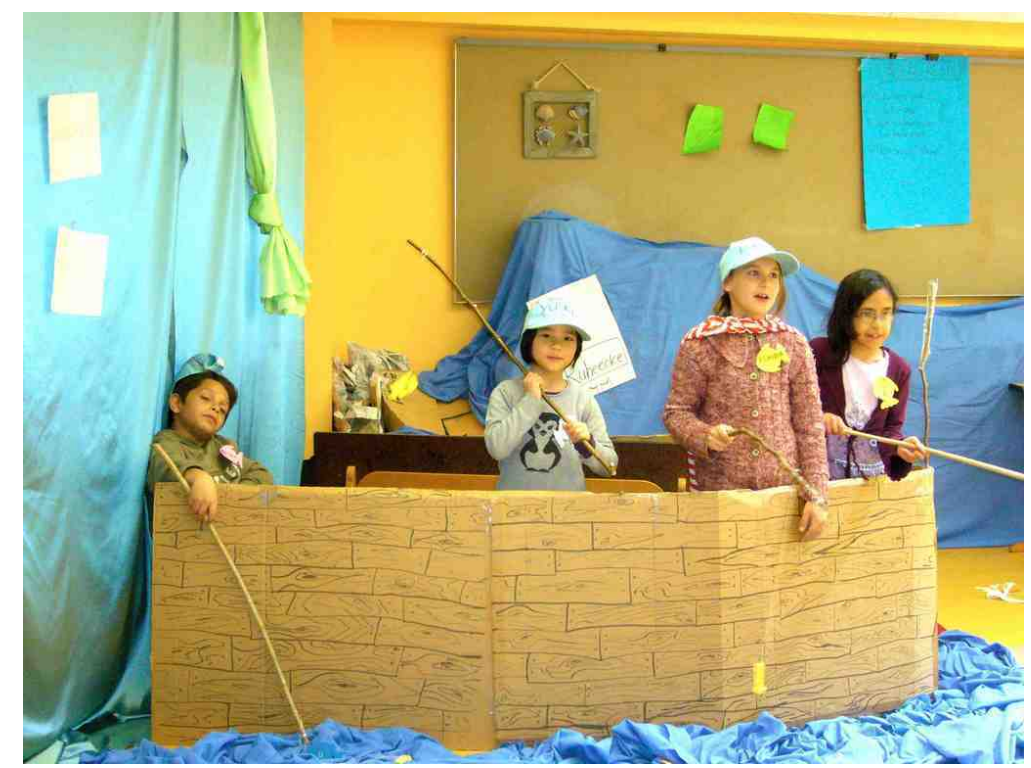

Abbildung 1: Gruppe der Fischer, Szenenerarbeitung mit selbstgebauten Requisiten, Tag 3

zentralen Merkmale des Tübinger Theatercamps. Ein (im Vergleich zu anderenorts stattfindenden Theaterprojekten) weiteres Herausstellungsmerkmal ist der sich am Sprachentwicklungsstand orientierende systematische Aufbau/Ausbau bildungssprachlicher Fähigkeiten in sprachlich relativ homogenen Gruppen (siehe 2.2).

\subsection{Dramagrammatische Sprachförderung im Theatercamp}

In Anlehnung an Evens Dramagrammtik (2003) wurde für das Tübinger Theatercamp ein Sprachförderkonzept entwickelt, das einerseits kindgerecht motivierend wirkt und andererseits versucht, den besonderen Ansprüchen von Kindern mit Deutsch als Zweitsprache (DaZ) gerecht zu werden (Bryant 2012). Unser Sprachförderkonzept führt (eingebunden in die Szenenerarbeitung für das Theaterstück) systematisch an die sprachlichen Anforderungen der Schule heran. Je nach Alter und sprachlicher Entwicklungsstufe werden im Camp bestimmte bildungssprachliche Bereiche in die Sprachförderung integriert und sukzessive aufgebaut. Bei den sprachschwächeren Kindern geht es u.a. um die präzise Verwendung von Lokalisierungsausdrücken und um die Versprachlichung temporaler Abfolgen, bei den Kindern der mittleren Sprachstufen wird u.a. an einer Erweiterung des Nebensatzspektrums und des Attributgebrauchs gearbeitet und bei den relativ fortgeschrittenen Kindern steht die Textarbeit (Textsorten, Textaufbau, Textkohäsion, ...) im Vordergrund. In den dramagrammatischen Einheiten, die immer am Vormittag des Camps stattfinden, liegt auf den Zielstrukturen ein besonderer Fokus. Die Szenen- und Rollenarbeit liefert den inhaltlichen Hintergrund für die Grammatikarbeit. Es kann am Ende der Einheit nur das von den Kindern erwartet werden, was vorher 


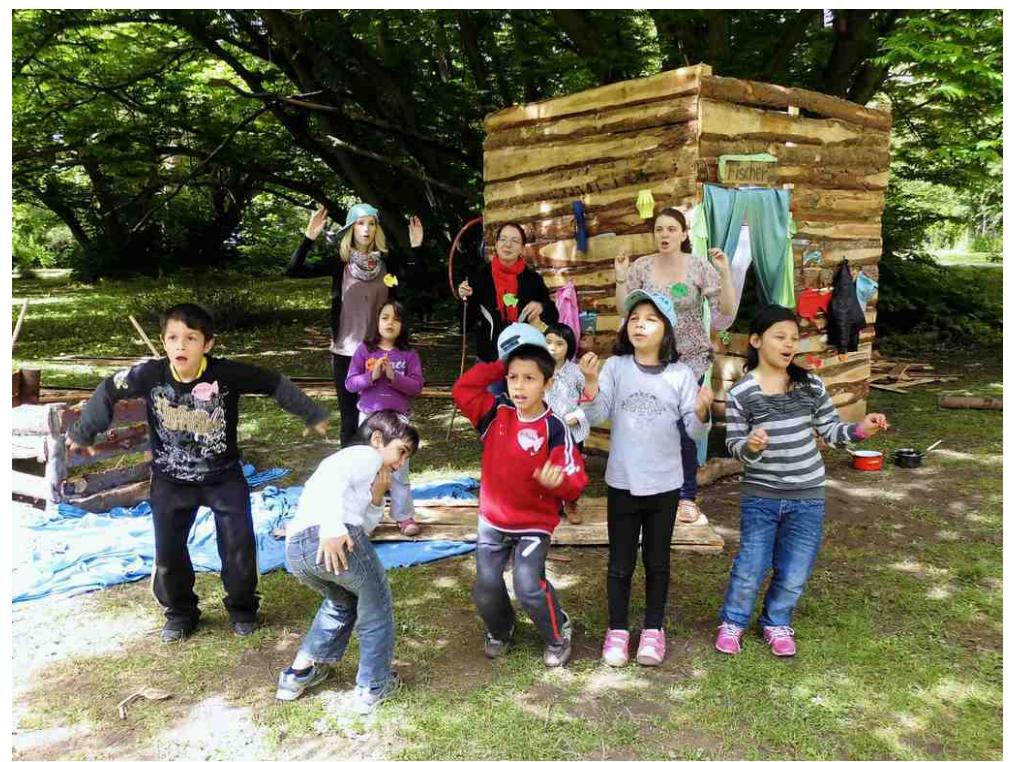

Abbildung 2: Gruppe der Fischer, Szenenerarbeitung vor selbstgebauter Hütte, Tag 6

in ausreichender Qualität und Quantität angeboten wurde. Bis die Kinder die Strukturen frei anwenden können, bedarf es zunächst eines rezeptiven Angebotes, einer metasprachlichen Reflexion sowie Strukturentlockungen (Elizitierungen) im kontrollierten Rahmen. Der weitere Weg hin zur freien Anwendung sollte binnendifferenzierend flankiert sein von sprachlichen Stützen (z.B. in Form ausgelegter Karteikarten), auf die die Kinder je nach Sprachsicherheit mehr oder weniger zugreifen.

Es liegen bereits einige wissenschaftliche Abschlussarbeiten vor, die die sprachmotivierende und sprachbildende Wirkung der Methode dokumentieren. Durch die Berücksichtigung des Sprachlevels bei der Gruppeneinteilung gelingt es, auch jene Kinder zum Sprechen, Lesen, Schreiben und Theaterspielen zu motivieren, die sich sprachbedingt in ihrer Schulklasse oft überfordert und ausgegrenzt fühlen.

Dramagrammatisches Phasenmodell — Eine Fördereinheit besteht in unserem Konzept abwechselnd aus fünf oder vier Phasen, und zwar in Abhängigkeit davon, ob eine bestimmte Struktur, Grammatikregel, Lesestrategie, etc. erarbeitet und explizit gemacht oder nur vertieft werden soll. Im erstgenannten Fall sind fünf Phasen vorgesehen: (1) Aufwärmphase, (2) Motivation, (3) Strukturvermittlung/Reflexion, (4) Strukturanwendung/Improvisation, (5) Präsentation/Reflexion.

In der Aufwärmphase werden Auflockerungs-, Assoziations-, Impuls- und Rotationsspiele durchgeführt, die einerseits dabei helfen, anzukommen und Hemmungen abzulegen und andererseits aber auch schon dazu dienen, bestimmte sprachliche Strukturen zu trainieren. Insbesondere Rotationsspiele 
erlauben es, sprachliche Konstruktionen in hoher Frequenz zu üben, was sich begünstigend auf den Erwerbsprozess auswirkt und den SchülerInnen erfahrungsgemäß großen Spaß macht, weil sie durch klare Vorgaben sprachliche Sicherheit erfahren und somit Kapazitäten freihaben, ihren Äußerungen eine emotional theatralische Farbe zu geben, was sich wiederum positiv auf die mentale Verankerung der Zielstrukturen auswirkt. Diese Phase, die Raum für Sprachspiele mit wiederkehrenden und leicht zu modifizierenden Konstruktionen gibt, eignet sich auch gut, um gezielt typische DaZ-Schwierigkeiten anzugehen und eventuell fossilisierte Sprachmuster zu überwinden. Inhaltlich haben die Sprachspiele der Aufwärmphase bereits einen Bezug zum übergeordneten Thema der Fördereinheit.

Die Motivationsphase dient u.a. dazu, eine die SchülerInnen begeisternde Aufgabe zu entwickeln, die sie unbedingt erfüllen wollen und für die sie auch bereit sind, ihr sprachliches Repertoire zu erweitern und sich auf Phase (3), die Strukturvermittlung/Reflexion, einzulassen. Idealerweise erarbeiten sich die SchülerInnen anhand eines speziell aufbereiteten Materials selbst die Zielstrukturen und reflektieren diese im Anschluss mit der Lehrkraft. Zum Beispiel könnten die zur Disposition stehenden Strukturen bestimmte morphologische Kontraste enthalten (wie beispielsweise mein Schal vs. meine Jacke oder sein Schal vs. ihr Schal), die zum Nachdenken über die Formunterschiede anregen. SprachlernerInnen suchen nach Form-Bedeutungszusammenhängen (Slobin 1973). Wenn sie Unterschiede in der Form bemerken, dann versuchen sie zu ergründen, welcher Bedeutungs- bzw. Funktionsunterschied damit einhergeht. Mit erstgenanntem Beispiel könnte man die LernerInnen darauf aufmerksam machen, dass possessive Artikelwörter hinsichtlich des grammatischen Geschlechts (Genus) mit dem Bezugsnomen kongruieren, im zweitgennannten Fall geht es um die Genuskongruenz mit dem Possessor. Grundschulkinder sind problemlos in der Lage, einen so transparent aufbereiteten Input metasprachlich zu reflektieren. Die Ergebnisse werden schriftlich (z.B. auf einem Plakat) festgehalten. Damit ist die Voraussetzung für Phase 4, für die Strukturanwendung/Improvisation, geschaffen. Dank der besprochenen und schriftlich fixierten, im Raum ausgelegten oder aufgehängten Hilfsstrukturen und Regeln sind die SchülerInnen nun in der Lage, die im Rahmen der Motivationsphase gestellte kommunikative Aufgabe zu erfüllen. Je nach inhaltlichem Thema wird in Abhängigkeit des Sprachstandes der Kinder und unter Berücksichtigung ihrer Erfahrungen mit dem dramapädagogischen Ansatz eine passende Inszenierungsform und Inszenierungstechnik ausgewählt, um die Strukturen nun anzuwenden. Die verschiedenen Inszenierungsformen lassen sich auf einem Kontinuum von stark gelenkt (u.a. Sprachlernspiele, Rollenspiele, Simulationen) bis weitgehend ungelenkt (u.a. szenische Improvisationen) anordnen. Mit Abnahme der lehrerseitigen Lenkung nimmt die Eigenverantwortlichkeit der LernerInnen zu (vgl. Even 2003: 156f.) Rollenspiele und Simulationen wenden wir erst an, wenn die Zielstrukturen wirklich verinnerlicht sind, d.h. in einer späteren Fördereinheit mit nur vier Phasen (ohne Strukturvermittlungsphase). Zur Gestaltung der Inszenierungsformen steht ein vielfältiges Repertoire an 
Inszenierungstechniken zur Verfügung. So eignen sich für Sprachhandlungen des Beschreibens und Interpretierens u.a. die Techniken Standbild, Mauerschau und Diashow, zur Anbahnung und Übung argumentativer Strukturen würde man hingegen Techniken wie Doppeln oder Spalier (vgl. Even 2003: 162f.) einsetzen.

Jede Fördereinheit endet mit einer Präsentation des Erarbeiteten und anschließender Reflexion, wobei darauf geachtet wird, dass bestimmte bildungssprachliche Antwortmuster zur Anwendung kommen. Zur Unterstützung liegen je nach Aufgabenstellung Satzbausteine aus (Ich bin der Meinung, dass ... / Besonders gut gefiel mir ...), die bei Bedarf genutzt werden können.

Gute Erfahrungen haben wir damit gemacht, die von zwei Förderkräften betreute Großgruppe (mit ca. zehn bis zwölf Kindern) für die Phase der Strukturanwendung/Improvisation zu teilen. Für die letzte Phase kommen die beiden Kleingruppen dann wieder zusammen und präsentieren sich gegenseitig das zuvor Erarbeitete. Die Aussicht auf eine kleine Präsentation setzt bei den Kindern enorme motivationale Kräfte frei, was sich wiederum positiv auf die Kreativität und Konzentrationsfähigkeit auswirkt.

Dramagrammatische Beispieleinheit zu Relativsätzen — Einerseits gehören Relativsätze in Schulbüchern zu den frequentesten Nebensatztypen, andererseits haben Kinder gerade mit diesen Konstruktionen enorme Schwierigkeiten, was sich nicht zuletzt auch an der Vermeidung dieser Strukturen erkennen lässt (Bryant 2015: 77). Im Theatercamp gehören Relativsätze daher zu den verstärkt geförderten Konstruktionen, und zwar in den sprachlich schon etwas fortgeschrittenen Gruppen, in denen die Kinder bereits andere Nebensatztypen mit Verbendstellung (wie z.B. Komplementsätze und Kausalsätze) verwenden. Die Schwierigkeiten, die LernerInnen mit deutschen Relativsatzkonstruktionen haben, sind syntaktischer und morphologischer Art (Bryant 2015: 81-85). In der hier dargestellten Einheit geht es um einfache Relativsatzkonstruktionen, die auch im Erstspracherwerb eine Vorreiterrolle spielen (Brandt et al. 2008; Bryant 2015). Hierbei bezieht sich das Relativpronomen auf die Nominalphrase eines vorangehenden Kopulasatzes. Bezugsnomen und Relativpronomen erscheinen beide im Nominativ.

Die Fördereinheit wurde für eine Gruppe konzipiert, der im zu erarbeitenden Theaterstück die Rolle der Regierung zukam. Einige der Kinder hatten aufgrund schlechter Schulerfahrungen eine starke Abwehrhaltung gegenüber dem Medium Schrift ausgebildet. Um das Interesse am Lesen und Schreiben wiederzubeleben, haben wir einen Präsidenten erdacht, der zwar selbst nie in Erscheinung trat, der aber mit den Regierungsmitgliedern im regen Schriftwechsel stand. So erhielten die MinisterInnen von ihm täglich einen Brief mit Informationen, Instruktionen und Fragen, den sie jeden Morgen aufgeregt erwarteten und wissbegierig lasen. Der Inhalt des präsidialen Briefes wurde oft so verfasst, dass sich daraus leicht die Motivation für die Zielstruktur ableiten ließ.

Ziel der Beispieleinheit war es, sich ,dem Volk' als Minister bzw. als Ministerin 
für einen bestimmten Zuständigkeitsbereich vorzustellen und dabei einen Relativsatz zu verwenden (Ich bin der/die Minister/Ministerin, der/die ...). In der Aufwärmphase wurde über den Input und über den gesteuerten Output die einfache Relativsatzkonstruktion bereits als Ankerstruktur gelegt. Für den eigenständigen produktiven Umgang mit der Struktur ist es jedoch wichtig, sich deren syntaktischen Aufbau noch einmal bewusst zu machen. Dies geschah in der Vermittlungsphase, in der die metasprachliche Reflexion angeregt werden sollte.

Nach diesen Hintergrundinformationen sei im Folgenden die Beispieleinheit, für die insgesamt zweieinhalb Stunden zur Verfügung standen, im Detail beschrieben.

\section{Aufwärmphase}

Zur Einstimmung wurden Bilder von Parlaments- und Regierungssitzungen an die Wand projiziert. Die Kinder übten sich im Nachstellen der Körperhaltungen und im Imitieren besonders markanter Gesichtsausdrücke. Im anschließenden Raumlauf stellten sie auf ein Stoppzeichen hin die von ihnen präferierte Mimik und Gestik dar. Die Übung wurde erweitert, indem sie dazu noch einen Laut oder ein Wort äußern konnten.

Nun bekam jedes Kind eine Karte mit einer Beschreibung, die auszuagieren war: Du bist Minister/Ministerin. Bei Versammlungen bist du oft unaufmerksam und spielst mit deinem Handy. Während sich die Kinder im Raum wie die beschriebenen Personen verhielten, fragte die FK: Wer ist der Minister, der bei Versammlungen oft einschläft? oder Wer ist die Ministerin, die bei Versammlungen oft einschläft? Gewünschte Antwort: Mehtap / sie (mit Zeigegeste) ist die Ministerin, die bei Versammlungen oft einschläft.

\section{Motivation}

Es klopfte und die Blitzpost-Rolle vom Präsidenten wurde hereingebracht. In dem Brief wies der Präsident daraufhin, dass es für die Regierungsmitglieder höchste Zeit sei, ihre Ministerposten einzunehmen. Es gäbe viel zu tun im Land und die Verantwortungsbereiche müssten geklärt werden. Damit keine Streitereien aufkämen, wer welches Ministerium übernähme, würde er ein Losverfahren vorschlagen. Zuvor sollten alle Regierungsmitglieder aber erst einmal gemeinsam überlegen, welche Ministerien zum Wohle der im CampLand lebenden Gruppen (Fischer, Handwerker, Forscher, ...) eingerichtet werden müssten. Er erwarte, dass die frisch gewählten MinisterInnen ihn schriftlich über ihren Verantwortungsbereich informieren und dass sie sich umgehend dem Volke präsentieren.

\section{Strukturvermittlung/metasprachliche Reflexion}

Nachdem die Ministerien in einem feierlichen Ritual ausgelost wurden, ging es darum, der präsidialen Anweisung zu folgen und eine kleine (ca. 2-3 Sätze umfassende) Vorstellung zu erarbeiten. Man diskutierte zunächst einzelne Vorschläge wie z.B. Ich bin die Handwerksministerin, Ich bin für das Handwerk zuständig oder auchIch bin die Ministerin, die für die Handwerker verantwortlich ist. Man kam überein, dass sich die jeweiligen Gruppen wohl eher mit der dritten Variante angesprochen fühlen. Es wurden die beiden geschlechtsspezifischen 
Relativsatzkonstrukionen Ich bin der Minister, der... und Ich bin die Ministerin, die .... einander gegenübergestellt. Die Schüler bemerkten, dass Artikel und Relativpronomen in ihrer Form übereinstimmen. Erst in späteren Einheiten würden sie erfahren, dass die Kongruenzbeziehung zwischen Bezugsnomen und Relativpronomen nur hinsichtlich Numerus und Genus gilt. Der Kasus wird bestimmt durch die syntaktische Funktion, die dem Relativpronomen im Relativsatz zukommt (vgl. Ich bin die Ministerin, der sie vertrauen können). Da viele DaZ-Kinder große Schwierigkeiten mit Genus und den verschiedenen Realisierungsvarianten (u.a. Relativpronomen) haben, war es an dieser Stelle der Förderung ausreichend, dass sie die Genuskongruenz (gut sichtbar bei Kasusübereinstimmung) zwischen Bezugsnomen und Relativpronomen erkennen und anwenden konnten.

\section{Strukturanwendung/Improvisation}

In der Kleingruppe schrieb nun jedes Kind seinen kurzen Vorstellungstext, derart: Mein Name ist Konstantin. Ich bin der Minister, der für die Drachenfischer zuständig ist. Je nach Sprachsicherheit konnte noch ein weiterer Satz hinzukommen. Die Förderkräfte hatten Erweiterungsbausteine vorbereitet. Die Kinder übten dann in der Kleingruppe ihren Auftritt am Rednerpult und setzten dabei die Mimik und Gestik ein, die sie in der Aufwärmphase für sich als PolitikerInnen entdeckt hatten.

\section{Präsentation/Reflexion}

Es folgte die Präsentation vor der großen Gruppe - vor dem Volk (siehe Abb. 3). Jede Vorstellung wurde mit einem tosenden Applaus und Begeisterungsrufen gewürdigt. Jede(r) MinisterIn durfte nach der Rede seinen / ihren Minitext unterschreiben und in die Blitzpost-Rolle für den Präsidenten stecken. Damit endete der dramapädagogische Prozess.

Im Anschluss wurde gemeinsam überlegt, ob und wenn ja, welche Aspekte der Einheit in das Theaterstück eingebaut werden sollten. Die SchülerInnen waren angehalten, ihre Entscheidungen zu begründen. Es lagen auch hierfür wieder Satzbausteine aus.

\section{Vom außerschulischen Theater-Feriencamp zur schulischen Theater-AG}

Nachdem sich das Tübinger Theatercamp in drei Durchläufen als Sprachförderkonzept bewährt hat, bestand die nächste Herausforderung darin, sich dem Nachhaltigkeitsproblem zu stellen. Es ist gelungen, die Stadt Tübingen davon zu überzeugen, dass es sich lohnt, auch in das im Folgenden vorzustellende Anschlussprojekt zu investieren. Die Stadt hat ihre finanzielle Unterstützung zunächst für zwei Jahre zugesagt. Über eine Verlängerung wird dann unter Berücksichtigung der Evaluationsergebnisse (siehe hierzu 4.4) neu zu verhandeln sein. 


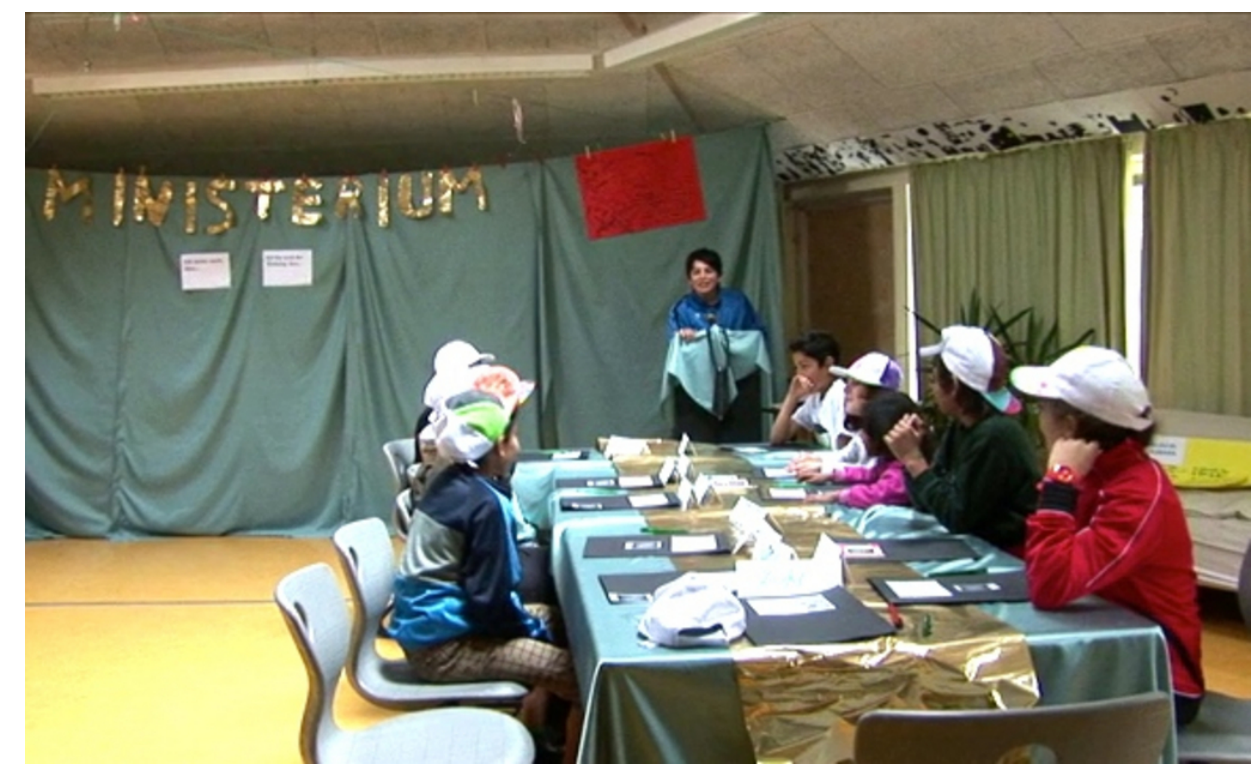

Abbildung 3: Präsentationsphase - Die Minister stellen sich vor

\subsection{Konzeptidee für die nachhaltige Förderung}

Um zu verhindern, dass sich die positiven Effekte des Theatercamps schon bald wieder verflüchtigen, erschien es den Verantwortlichen ausgesprochen wichtig, dem Camp eine Maßnahme folgen zu lassen, die

a) möglichst viele der Kinder aus dem Sprachcamp einschließt,

b) methodisch und personell hieran anknüpft,

c) sich gut in den Schulalltag integrieren lässt,

d) einen längeren Förderzeitraum umfasst.

Zum Theater-Feriencamp kommen die Kinder aus allen Stadtteilen in die Innenstadt und müssen dabei zum Teil weite Wege zurücklegen. Sie werden vormittags von den Eltern gebracht und am Nachmittag wieder abgeholt. Um die dramapädagogische Sprachförderarbeit nach dem impulsgebenden und die Kinder begeisternden Ferienprojekt fortzuführen, wurden im Anschluss an das Camp 2015 an drei Schulen Theater-AGs etabliert, und zwar an drei zentralen Standorten in der Innen- und Nordstadt. Damit wird der Bedingung (a) entsprochen, da die Kinder je nach Wohn- bzw. Schulstandortnähe eines der drei AG-Angebote wahrnehmen können.

Wie in (b) gefordert, wird im Rahmen der Theater-AGs methodisch in gleicher Weise vorgegangen wie im Camp. Auch hier werden Phasen der expliziten Sprachvermittlung und Reflexion in den dramapädagogischen Prozess integriert. Einen Unterschied gibt es hingegen hinsichtlich der thematischinhaltlichen Vorgaben. Während diese sich im Camp aus dem gemeinsam zu erarbeitenden und aufzuführenden Theaterstück ergeben, soll die Arbeit in der Theater-AG nicht auf eine große Theaterproduktion hinauslaufen. Stattdessen sollen die vielfältigen Möglichkeiten der Dramapädagogik genutzt werden, um konkrete Fachinhalte handlungsbegleitend sprachlich zu erarbeiten und zu 
inszenieren (siehe hierzu 3.2 und 4).

Durchgeführt werden die Theater-AGs von einer sprachwissenschaftlich und theaterpädagogisch erfahrenen Doktorandin und einer DaZ-Studierenden. Beide waren im Camp in die dramagrammatische Sprachförderung eingebunden. Somit ist die methodische und personelle Kontinuität des CampAnschlussprojekts, vgl. (b), garantiert.

Dem Punkt (b) entsprechend sind die Theater-AGs in allen drei Schulen gut im Nachmittagsband integriert und finden zeitgleich mit anderen AGs statt. Sie werden von den jeweiligen Schulen als Bereicherung angesehen und entsprechend unterstützt.

In der letzten Bedingung (d) geht es um den Förderzeitraum. Die Finanzierung des Projekts ist zunächst auf zwei Jahre begrenzt. Da die AGs in der Grundschule angesiedelt sind, können die Kinder, die zum Zeitpunkt des Camps in Klasse 4 waren, nach dem Wechsel in die Sekundarstufe I leider nicht mehr von dem Angebot profitieren. Die Kinder, die in den Pfingstferien 2015 die Klasse 2 besuchten, können nach dem Camp zwei Jahre gefördert werden, die Kinder der Klasse 3 mindestens ein Jahr. In einem solchen Förderzeitraum ist es möglich, systematisch die Sprachkompetenz aufzubauen und die GrundschülerInnen auf die bildungssprachlichen Anforderungen der Sekundarstufe I vorzubereiten. Erfahrungsgemäß stoßen Maßnahmen, die sich über einen längeren Zeitraum erstrecken und den SchülerInnen durch die gewährleistete Kontinuität wirkliche Entfaltungsmöglichkeiten bieten, auch bei den LehrerInnen auf große Akzeptanz und Unterstützungsbereitschaft. Diese Unterstützung ist von zentraler Bedeutung für das Gelingen des Projekts. Nur wenn sich die LehrerInnen dafür begeistern können, werden sie sich im überlasteten Schulalltag die Zeit dafür nehmen, mit uns über die zu fördernden Kinder zu sprechen und sich mit uns über potentielle Fachthemen für die AG abzustimmen. Nur dann werden sie ein Auge darauf haben, dass die Kinder auch bei der AG erscheinen und sich mit ihnen darüber austauschen wollen.

\subsection{Motivation für die fachsensible Sprachförderung}

Der Einsatz dramapädagogischer Elemente zur Vermittlung schulischer Fachinhalte ist nicht ganz neu. Das methodische Konzept Drama in Education, das sich wissenschaftstheoretisch auf reformpädagogische Traditionen und kognitive Handlungstheorien stützt, ist in England ,seit Mitte des 20. Jahrhunderts sowohl als eigenständiges Fach als auch als übergreifendes Lehrprinzip etabliert" (Bonnet \& Küppers 2011: 42). Britischen Autoren zufolge (u.a. Bolton 1984), verschafft der dramapädagogische Unterricht differenziertere Einsichten, unterstützt die Sprachentwicklung, fördert die Wissensfindung und durch die affektive Komponente auch die nachhaltige Wissensverankerung (Even 2003: 146). Für die Einbindung drama- und theaterpädagogischer Elemente in den Schulunterricht plädiert auch das Konsortium der internationalen DICE-Studie, an der ca. 5000 SchülerInnen aus 12 Ländern teilnahmen, und in der sich positive Effekte in den untersuchten Kompetenzbereichen (communication in 
the mother tongue; learning to learn; interpersonal, intercultural and social competences; entrepreneurship; cultural expression) feststellen ließen (vgl. DICE Konsortium 2010).

Bei unserem Ansatz geht es zwar auch um fachliche Inhalte, aber nicht um den eigentlichen Fachunterricht, sondern um eine zusätzliche Maßnahme für förderbedürftige Kinder. Zum besseren Verständnis, wo sich diese im Schulalltag im Kontext ähnlicher Angebote verorten lässt, hier ein kurzer Überblick verwandter Begrifflichkeiten, wie sie in der sprachdidaktischen Literatur Verwendung finden. Es wird unterschieden zwischen (a) sprachsensiblem Fachunterricht, (b) fachsensiblem Sprachunterricht und (c) fachsensibler Sprachförderung. Bei (a) bemüht sich die Lehrkraft im Fachunterricht (z.B. Biologie, Mathematik, Geschichte) auf die sprachlich heterogene Schülerschaft einzugehen, in dem nicht nur die Inhalte sondern parallel auch fach- und bildungssprachliche Phänomene in den Blick genommen werden. Mit (b) ist der Deutschunterricht gemeint, der in Bezug auf die sprachlichen Anforderungen der Schule und deren Vermittlung mit anderen Fächern kooperiert und beispielsweise in fachspezifische Textsorten (z.B. Protokoll) einführt. Bei (c) handelt es sich um ein zusätzliches Angebot außerhalb der Unterrichtszeit für jene SchülerInnen, deren sprachliche Fähigkeiten noch nicht hinreichend entwickelt sind, um dem Unterricht folgen zu können. Üblicherweise beginnt die Beschäftigung mit einem Thema in der umgangssprachlichen Varietät. Man spricht über in der aktuellen Situation Wahrnehmbares. In der weiteren Beschäftigung mit dem Thema wird die Sprache zunehmend komplexer, sie wird lexikalisch präziser und strukturell elaborierter. Die SchülerInnen sind nun in der Lage, einen bildungssprachlichen Text zu verfassen oder einen bildungssprachlichen Vortrag über das Thema zu halten. Idealerweise wird ein Unterrichtsthema über einen Zeitraum von mehreren Sitzungen vorbereitet und nicht etwa nachbereitet. Nur dann erleben die Kinder einen sofortigen Erfolg, indem sie sich nämlich erstmals aktiv am Unterrichtsgeschehen beteiligen können, was sich wiederum positiv auf das Selbstwertgefühl auswirkt und die Lernmotivation steigert.

Auch wenn die fachsensible Sprachförderung nur in Bezug auf ein bestimmtes Fach erfolgt, ist dennoch davon auszugehen, dass die anderen Fächer von der Intervention profitieren, denn schließlich sind es neben fachspezifischen vor allem die bildungssprachlichen Kompetenzen, die sich entwickeln und die fachübergreifend relevant sind.

Das in Tübingen aktuell in der Bewährungsphase befindliche und hier vorzustellende Konzept ist ein spezifischer Fall von (c). Es knüpft an das in England fachübergreifend etablierte Lehrprinzip Drama in Education an und verbindet dieses mit zentralen Ideen der dramapädagogischen Fremd/Zweitsprachförderung (u.a. Schewe 1993; Even 2003; Bryant 2012) und macht sie für das kombinierte Fach- und Sprachlernen nutzbar. 


\subsection{Berücksichtigung der Operatoren}

In unserem Förderkonzept kommt den sogenannten Operatoren eine zentrale Bedeutung zu. Es handelt sich hierbei um Aufgabenstellungen, die in Form handlungsinitiierender Verben (z.B. beschreiben, erklären, begründen) gegeben werden und deren Ausführung Basis der Leistungsbewertung im Fachunterricht ist. Sprachschwache SchülerInnen haben bereits mit der Dekodierung dieser Anweisungen Schwierigkeiten. Doch selbst wenn die Bedeutung des Operators klar wäre, bräuchte es immer noch die relevanten Strukturen und Antwortmuster und diese speisen sich aus dem für viele SchülerInnen nur schwer zugänglichen Repertoire der schriftnahen Bildungssprache. Daher soll auch in unserem Projekt der Gebrauch ausgewählter Operatoren gezielt gefördert werden. Hierfür arbeiten wir mit wiederkehrenden Strukturbausteinen, die ausgelegt bzw. aufgehängt werden. Die SchülerInnen, die selbst nicht in der Lage sind, solche komplexen Konstruktionen zu formulieren, haben so die Möglichkeit, bestimmte Antwortmuster als Chunk (als „Brocken“) zu lernen. Auch wenn die vorgegebenen Einheiten für die LernerInnen (zumindest partiell) noch unanalysiert sind, wissen sie doch um die Kontexte, in denen diese Verwendung finden. Das gibt Sicherheit und eröffnet Kapazitäten für andere Sprachlernaufgaben. Zudem gewinnt die Sprache durch Chunks an Flüssigkeit und Natürlichkeit (Stengers et al. 2011). Auch wir MuttersprachlerInnen gebrauchen ständig als feste Brocken memorisierte Einheiten (u.a. Pawley \& Syder 1983; Erman \& Warren 2000). Durch den Gebrauch solcher Chunks, die wir als Ganzes abrufen und in unsere Rede einfügen können, gewinnen wir Zeit, um zu überlegen, was inhaltlich noch zu sagen wäre (Handwerker \& Madlener 2009: 6). Die Operatorenbausteine bieten eine hervorragende Gelegenheit zum Erlernen von Chunks und können durch leichte Modifikation bei den wiederkehrenden Antwortmustern auch die sprachlichen Analyseprozesse anregen bzw. beschleunigen (ibid. 6).

\section{Methodisch-inhaltlicher Vergleich der Theater-AGs in Hamburg und Tübingen}

Während sich in Evaluationen direkt im Anschluss an die Feriencamps positive Effekte feststellen lassen, zeigen Follow-Up-Tests, die einige Monate später durchgeführt werden, hingegen kaum noch Vorsprünge der CampteilnehmerInnen gegenüber den MitschülerInnen in Kontrollgruppen (vgl. Kinze 2012: 94). Den Camps ist damit ein Nachhaltigkeitsproblem zu attestieren (vgl. Kinze 2012: 85; Sting 2012: 62; Bryant 2012: 28), dem sowohl Hamburg als auch Tübingen in Form von Theater-AGs begegnen.

Hamburg hat bereits 2012 damit begonnen, entsprechende AGs einzurichten. Tübingen ist dem 2015 gefolgt. An beiden Orten finden die AGs wöchentlich an einem Nachmittag für 90 Minuten statt.

Trotz vieler Gemeinsamkeiten in Anspruch und Konzeption einer dem Camp folgenden Maßnahme gibt es doch auch - wie in Tabelle 1 dargestellt - einige 
Unterschiede zwischen den AG-Angeboten beider Standorte.

Tabelle 1: Vergleich der Theater-AGs in Hamburg und Tübingen

\begin{tabular}{|c|c|c|}
\hline & $\begin{array}{l}\text { TheaterSprachKurse } \\
\text { Hamburg }\end{array}$ & $\begin{array}{l}\text { Theater-AGs } \\
\text { Tübingen }\end{array}$ \\
\hline Methode & $\begin{array}{l}\text { Neunteiliges } \\
\text { Bausteinsystem }\end{array}$ & $\begin{array}{l}\text { Dramagrammatisches } \\
\text { Fünf-Phasen-Modell }\end{array}$ \\
\hline Themenbasis & Kinderliteratur & Sachkunde (MeNuK) \\
\hline Förderschwerpunkte & $\begin{array}{l}\text { Lesekompetenz, } \\
\text { Lesevergnügen, } \\
\text { Grammatik des Verbs }\end{array}$ & $\begin{array}{l}\text { Auf- und Ausbau } \\
\text { der Bildungssprache } \\
\text { in Verknüpfung mit Fachthemen } \\
\text { unter Berücksichtigung DaZ- } \\
\text { spezifischer Schwierigkeiten }\end{array}$ \\
\hline $\begin{array}{l}\text { Umgang mit } \\
\text { Mehrsprachigkeit }\end{array}$ & Portfolio, Sprachspiele & Sprachspiele \\
\hline
\end{tabular}

An beiden Standorten wird in den AGs methodisch in gleicher Weise wie im Camp vorgegangen. Während in Tübingen die fünf- bzw. vierphasigen dramagrammatischen Einheiten den AG-Ablauf (siehe 2.2.1) bestimmen, nutzt man in Hamburg ein variables Bausteinsystem. Es besteht aus neun verschiedenen Bausteinen: Begrüßen, Vorlesen, Erinnern - Wiederholen - Festigen, Spielen Singen - Bewegen - Entspannen, Grammatik erforschen, Reflektieren - Dokumentieren, Lesestrategien - Leseübungen, Szenen entwickeln - Verabschieden (vgl. Neumann et al. 2012: 8). Diese Bausteine werden schrittweise in die TheaterSprachKurse eingeführt und tauchen dann ritualisiert immer wieder in bestimmten Phasen der Kurse auf. Es müssen nicht alle Bausteine an einem Kurstag aufeinander folgen, jedoch bilden in Hamburg der erste und der letzte Baustein den Rahmen jeder Einheit (vgl. Neumann et. al. 2012: 8). Das rhythmisierte Nutzen der Bausteine soll dazu führen, dass „sich Spracharbeit und Theaterarbeit gegenseitig durchdringen" (ibid. 9). Wie sich die Bausteine zusammensetzen, entscheidet die Fokussierung einer Kurseinheit. Sie liegt entweder auf dem Lesen, der Grammatikförderung (insbesondere Tempusformen des Verbs, Modalverben, reflexive Verben, trennbare Verben), dem Umgang mit Mehrsprachigkeit oder auf der Theaterarbeit (vgl. ibid. 20).

Wie auch in Tübingen steht in Hamburg die Bildungssprache in Abgrenzung zur Alltagssprache im Mittelpunkt (vgl. ibid. 15f.). Trotzdem unterscheidet sich die Schwerpunktsetzung der Standorte. Das Lesen nimmt in Tübingen weniger Raum ein als in Hamburg. Der Fokus liegt hier eher auf der mündlichen und schriftlichen Ausdrucksfähigkeit und der Vermittlung der hierfür relevanten Strukturen. Es geht in Tübingen um den Auf- und Ausbau konkreter bildungssprachlicher Fähigkeiten im Fachkontext unter Berücksichtigung DaZ-spezifischer Erwerbsschwierigkeiten (u.a. Nominalflexion, Lokalisierung, 
Nebensatzspektrum). Aus diesem Grund ziehen wir die thematische Basis nicht wie in Hamburg aus Kinderbüchern sondern aus dem Fächerverbund MeNuK (Mensch, Natur, Kultur).

\section{Konkrete Umsetzung der fachsensiblen dramagrammatischen Sprachförderung in Theater-AGs}

Die 35 der über 60 Kinder aus dem Camp, die auch nach den Ferien noch an der Grundschule sind, wurden von einer der beiden AG-leitenden Förderkräfte an ihren Schulen besucht und persönlich gefragt, ob sie an einer Theater-AG im Anschluss an das Camp teilnehmen wollen. Die Reaktionen waren weitgehend positiv, sodass nun mit 29 Kindern die Mehrheit der infrage kommenden Kinder an den AGs teilnimmt.

Drei Tübinger Grundschulen in verschiedenen Bezirken wurden für die AGs ausgewählt. Neben den Anmeldezahlen und der Erreichbarkeit für die Kinder, die aus nahegelegenen Grundschulen zur AG-Grundschule kommen, spielte auch der Sprachstand bei der AG-Bildung eine Rolle. An zwei der drei Standorte verfügen die acht bis zehn Kinder der AG über ähnliche sprachliche Fähigkeiten. Am dritten Standort war diese Aufteilung nicht möglich. Hier ist es notwendig, die SchülerInnen nach Aufgabenart und sprachlichen Anforderungen zu trennen, um ihnen eine optimale Förderung zukommen zu lassen.

Um der Präsentierfreude der Kinder gerecht zu werden, endet jeder Theaternachmittag in den AGs mit der Präsentationsphase auf der Bühne gefolgt von großem Applaus und der Reflexionsrunde. Die Erfahrung aus den Camps zeigt, dass die meisten Kinder überdies sehr gern vor einem größeren Publikum auftreten. Wegen der immer gleichen Übungsabläufe unmittelbar vor der Aufführung weicht jedoch oftmals die anfängliche Euphorie und wandelt sich in einen gewissen Übungsverdruss. Aus diesem Grund soll in Absprache mit den Schulen und Lehrkräften ein kleinerer Rahmen gefunden werden, in dem eine kurze, wenig aufwendige Präsentation möglich ist. Die Zeit und den Übungsaufwand, den es für den letzten Schliff einer perfekten und großen Aufführung bräuchte, investieren wir stattdessen in der AG, um an der sprachlichen Progression zu arbeiten.

Ziel hierbei ist es, im Fachkontext auf die bildungssprachlichen Anforderungen der Sekundarstufe I vorzubereiten. Die LehrerInnen des Fachs MeNuK (Mensch, Natur, Kultur) wurden deshalb nach Themengebieten gefragt, die sie aufgrund ihrer Komplexität zur Vorentlastung in den AGs empfehlen würden. Genannt wurden u.a.: Reporter/Zeitung, Tiere, Fahrrad/Straßenverkehr, Wetter/Klima, Gesundheit/Körper, Abfall/Umwelt. Diese Vorschläge bilden gemeinsam mit den sich aus dem Sprachstand der Kinder ableitenden Förderschwerpunkten die Grundlage für die dramagrammatischen Fördereinheiten. 


\subsection{Verknüpfung von Sprache, Fach und Drama - Eine Herausforderung}

Der Baden-Württembergische Bildungsplan für Grundschulen fordert eine Schnittstelle zwischen sprachlichen, inhaltsbezogenen und handlungsorientierten Fächern zu schaffen, weil „[d]ie Vermittlung von Weltwissen, sei es in der Mutter- oder Zielsprache, [... ] immer auch mit dem Auf- und Ausbau von bestimmten Teilen der Sprachkompetenz verbunden [ist]“ (Bildungsplan BW 2004: 68).

Werden (Fremd-) Sprachenunterricht und andere Fächer der Grundschule verbunden, können „Kinder ihr Welt- und Handlungswissen zugleich in der Zielsprache und in Sachgebieten [erweitern]" (ebd.). Dazu eignet sich in der Grundschule im besonderen Maße der Fächerverbund MeNuK (Mensch, Natur, Kultur), denn die „Auseinandersetzung mit Natur und Kultur regt zu gedanklicher Durchdringung, zu unterschiedlichen Darstellungsweisen und zu eigenen kreativen Prozessen an und führt so zu einer grundlegenden Bildung“" (ibid. 96).

Obgleich Eltern und Lehrkräfte Fächerverbünden gemeinhin kritisch gegenüberstehen (Stellungnahmen des Landeselternbeirates Baden-Württemberg 2014, Landesinstitut für Schulentwicklung: Abschlussbericht Evaluation der Fächerverbünde Baden-Württemberg 2011: 36), spricht man dem Fach MeNuK allerdings eine Eignung zur Vernetzung von Themen zu (ibid. 66). Jedoch herrscht unter Lehrkräften eine generelle Unsicherheit, wie Inhalte in Fächerverbünden zu verknüpfen seien (ibid. 43f.).

Der AG-Rahmen schafft hier Möglichkeiten, im Austausch mit den Lehrkräften entsprechende Ideen zu generieren und auszuprobieren. Wie eine Verknüpfung recht unterschiedlicher Fachthemen in Verbindung mit der Förderung bildungssprachlicher Aspekte gelingen kann, soll im Folgenden kurz skizziert werden. Exemplarisch hierfür steht das Vorgehen in der sprachlich am fortgeschrittensten Theatergruppe, in der sich die Kinder hauptsächlich in ihren schriftsprachlichen Kompetenzen von ihren monolingualen MitschülerInnen unterscheiden. Die Kinder dieser sprachlich stärkeren AG-Gruppe erhalten im Rahmen der dramagrammatischen Einheiten Unterstützung auf drei Ebenen:

- sprachstrukturelle Ebene,

- fachinhaltsbezogene Ebene,

- fachsprachlich-handlungsinitiierende Ebene (Operatoren als fachbezogene Sprachhandlungsinstruktionen).

Abbildung 4 gibt einen ersten Eindruck davon, wie diese drei verschiedenen Ebenen über den Zeitraum eines Schuljahres verbunden werden können:

Während die Kinder in die Rolle von ReporterInnen treten (fachinhaltsbezogene Ebene-Mitte) und ihre eigene Reporterpersönlichkeit ausbilden, erhalten sie verschiedene Aufträge. Zur Ausbildung des Rollencharakters sollen Beschreibungen angefertigt werden, die gleichzeitig das Beschäftigen mit dem Operator 


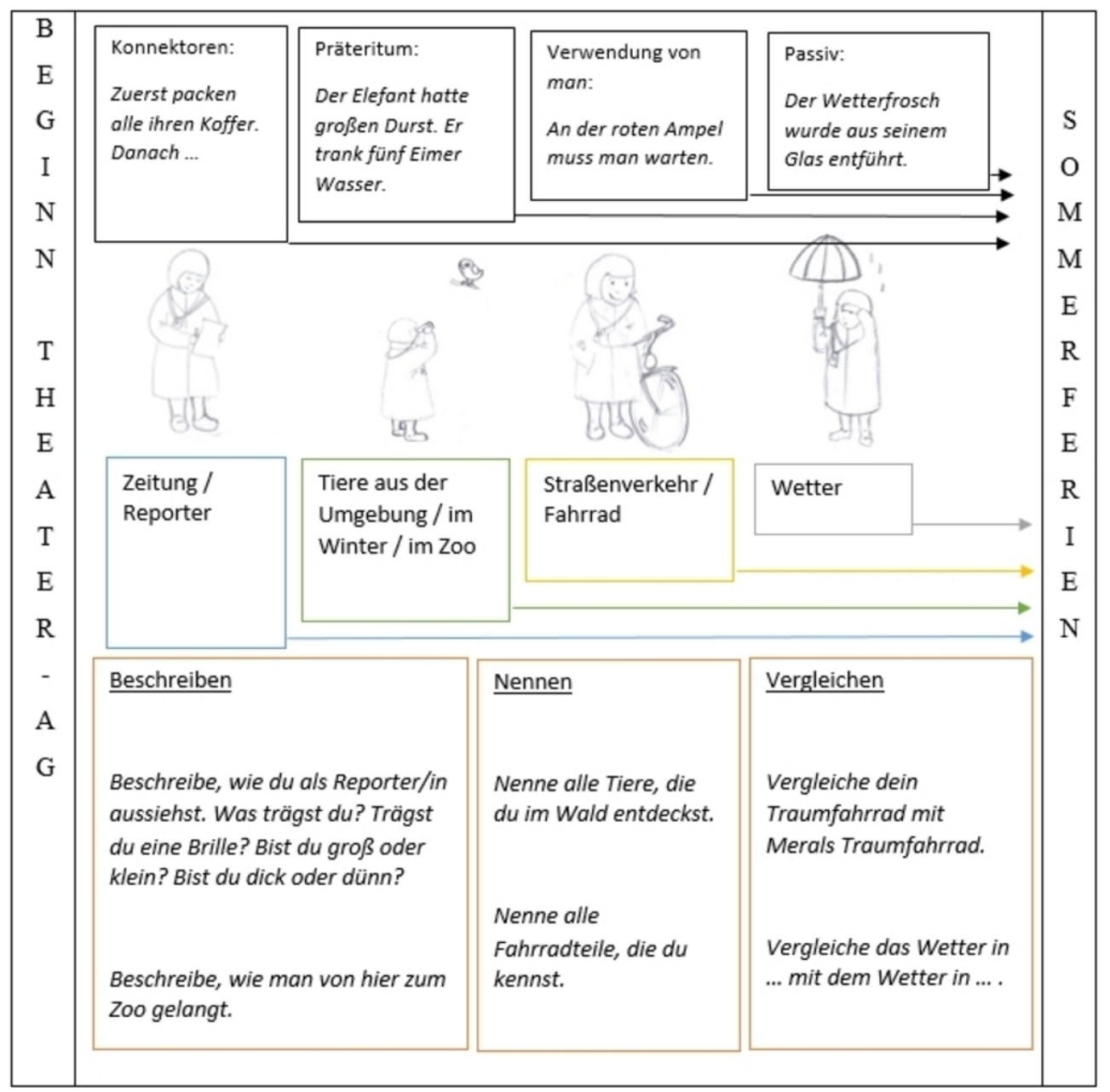

Abbildung 4: Drei Verknüpfungsebenen - sprachstrukturell (oben), fachinhaltsbezogen (Mitte) und fachsprachlich-handlungsinitierend (unten)

beschreiben verlangen (fachsprachlich-handlungsinitiierende Ebene - unten). Dazu tragen die Kinder in den ersten Sitzungen zusammen, wo ein Reporter arbeiten kann - zum Beispiel bei der Zeitung - und was seine Tätigkeitsfelder sind. Schrittweise wird dazu eine eigene Reporterpersönlichkeit entwickelt, mit der die Kinder sich im weiteren Verlauf der AG identifizieren können. Wie sehen sie aus, was mögen sie, wie verhalten sie sich, worüber berichten sie gern? Jedes Kind erstellt dazu seinen eigenen Presseausweis. Verschiedene Techniken, die das Ausdrücken unterschiedlicher Emotionen erproben, Pantomimespiele und Raumläufe bieten sich in diesen Einheiten als Standardübungen an, um den „Zusammenhang zwischen Gefühl und Körper auszubilden“ (Lechthaler 2004: 62).

Die Rollenpersönlichkeit beibehaltend müssen verschiedene Aufgaben erledigt werden, häufig in schriftlicher Form. So sollen beispielsweise Ablaufpläne für einen Reiseantritt angefertigt werden, wobei der Fokus der Fördereinheit 
auf den Auf- und Ausbau der Konnektoren gelegt wird (sprachstrukturelle Ebene - oben). Um der Arbeit als ReporterIn adäquat nachkommen zu können, wird den Kindern ein Reporter-Handwerkskoffer zur Verfügung gestellt, dessen Inhalt schrittweise zusammen mit den Förderkräften entdeckt wird. Es finden sich zahlreiche Tipps und Anregungen für die Reporterarbeit darin. Sie geben Anlass, um über die sprachstrukturellen Förderpunkte (Ebene oben) nachzudenken und zu diskutieren. So kann der Koffer Umfragebögen enthalten, die ermitteln, ob die Kinder als ReporterInnen bei ihren Berichten das Perfekt oder das Präteritum bevorzugen. Die Fragebögen sind mit Beispielsätzen gestaltet. Außerdem liegen Kommunikationskarten aus, um den Kindern mögliche redeeinleitende Antwortmuster vorzuschlagen. Die Förderkräfte lenken dabei die Diskussion als teacher in role. Eingebettet wird ein solches Beschäftigen mit sprachlichen Strukturen in das Szenario einer Redaktionssitzung. Damit bleibt die Verknüpfung zur fachinhaltsbezogenen Ebene durchgängig bestehen.

Von einer erdachten Chefredaktion erhalten die Reporter-Kinder weitere spannende Instruktionen per Mail oder Brief. Mal sind Protokolle, mal Beschreibungen, mal Berichte, mal Vergleiche gefordert. Hierbei geht es neben der Einbindung sprachstruktureller Förderaspekte (Ebene oben) darum, die Verwendung verschiedener Operatoren zu motivieren (Ebene unten) und darum, auf der fachinhaltsbezogenen Ebene (Mitte) für Abwechslung zu sorgen. Zum Beispiel sollen die ReporterInnen für eine Sonderausgabe der Zeitung über Tiere schreiben. Die Kinder können hierbei abwechselnd auch einmal in die Rolle eines Tieres schlüpfen. Für jede der Auftragsbearbeitungen wird mit unterschiedlichen Übungsformaten gearbeitet, in denen die Kinder ihre Vorstellungskraft verbessern und ihre darstellerischen Fähigkeiten weiterentwickeln, beispielsweise bei der Erstellung kleiner Szenen oder in Improvisationen.

Nachdem in mehreren Einheiten über Tiere des Zoos berichtet wurde, kann es im Anschluss daran um Waldtiere gehen. Dazu stellt sich beispielsweise die Frage, wie die Kinder in ihren Reporterrollen von den Redaktionsräumen zu den Tieren im Wald gelangen (fachinhaltsbezogene Ebene). Besprochen werden kann, welches Fahrzeug sich dafür eignet. Es muss leise genug sein, um die Tiere nicht zu erschrecken. Hier bietet sich die Überleitung zum Thema Fahrrad an. Die Kinder können dazu ihre Traumfahrräder malen und beschreiben (Operatorenebene). Alle Fahrradteile müssen dafür benannt werden (Operatorenebene). Es kann später gefragt werden, ob das Traumfahrrad denn auch verkehrssicher ist und wie man sich im Straßenverkehr verhält (fachinhaltsbezogene Ebene). Auch lassen sich die unterschiedlichen Traumfahrräder miteinander vergleichen (Operatorenebene).

Dargestellt wurde für den Zeitraum von einem Schuljahr in nur groben Zügen, wie eine Verknüpfung von Sprache, Fach und Drama gelingen kann. Eingetaucht in die Reporter-Rolle und im regen Austausch mit der Chefredaktion setzen sich die Kinder permanent mit Sprache auseinander und entwickeln den Ehrgeiz, ihr Ausdrucksrepertoire stetig zu erweitern. Gleichzeitig erschließen sie sich fachthematische Bereiche, die dann mit sprachhandlungsinitiierenden Operatoren bearbeitet werden. Zur Unterstützung ausliegende Satzbausteine, 
metasprachliche Reflexionen, ein hohes Maß an Wiederholungen sowie eine dem Entwicklungsstand angemessene, langsam steigende Sprachkomplexität schaffen ein Gefühl von sprachlicher Sicherheit und damit auch Raum für sprachliche Kreativität. Da die Themen miteinander in Bezug stehen, können die Kinder auch inhaltlich immer auf schon Vertrautem aufbauen und sich so im Gespräch und im Spiel mit ihrem Vorwissen einbringen. Auch das verschafft ihnen Erfolgserlebnisse und erhöht die Motivation, dabei zu bleiben.

\subsection{Dramagrammatische Beispieleinheit mit Fachbezug}

Während im vorhergehenden Abschnitt ein ganzer Jahresplan skizziert wurde, und zwar für sprachlich relativ weit fortgeschrittene Kinder, soll in diesem Abschnitt nun eine einzelne Fördereinheit dargestellt werden, die sich an sprachschwächere Kinder richtet. Diese Kinder sind zwar schon in der Lage einfache Sätze zu produzieren, sie haben aber u.a. noch Schwierigkeiten im Bereich der Genus- und Kasuszuweisung.

Zur Einordnung der Beispieleinheit in die sprachstrukturelle Progression im Rahmen der AG-Arbeit: In vorausgehenden Einheiten haben sich die Kinder bereits mit den Formunterschieden bei possessiven Artikelwörtern der ersten und zweiten Person beschäftigt. Anhand von Kontrastpaaren (mein Schal vs. meine Jacke) haben sie entdecken können, dass zwischen possessivem Artikel und Bezugsnomen hinsichtlich Genus und Numerus eine Übereinstimmung besteht. Die Einheiten sind so aufgebaut, dass erst in späteren Schritten Kasusunterschiede der possessiven Artikelwörter von Bedeutung sind. Zunächst einmal geht es nur um die Verwendung im Nominativ. Die folgende Einheit führt an possessive Artikel der dritten Person heran. Die Kinder sollen bemerken, dass sie hier auf die Genuskongruenz mit dem Possessor (sein Schal vs. ihr Schal) achten müssen. Es ist nicht zu erwarten, dass nach einer ersten Einheit die possessiven Artikelwörter der dritten Person schon internalisiert sind und produktiv verwendet werden können. Deshalb enthält die nun skizzierte AG-Stunde viele rezeptive Anteile. Die Sprachproduktion rückt in den Folgeeinheiten dann zunehmend in den Vordergrund.

Zudem wird in dieser Einheit auf der fachinhaltsbezogenen Ebene das neue MeNuK-Thema Klima eingeführt. Die SchülerInnen sollen zunächst dafür sensibilisiert werden, dass sich das Wetter und Klima in den vielen Ländern der Erde unterscheidet. In späteren AG-Stunden können anhand der an diesem Theaternachmittag gewonnenen Erkenntnisse Klimazonen und der Klimawandel thematisiert werden.

Bereits in der Aufwärmphase wird sowohl auf den Fachinhalt der Stunde (verschiedenes Klima) als auch auf die zu fördernde Struktur (possessive Artikelwörter der dritten Person) eingegangen. Nach einem ritualisierten Begrüßungsrap schließt sich wie gewohnt eine Wachklopfrunde an. Nachdem ein paar Mal in der aus vorigen Einheiten bekannten Struktur gefragt wurde: Was soll wachwerden? Dein Bein? Deine Hände? Und mehrmals -von Kindern und der zweiten Förderkraft im Raum - geantwortet wurde: Nein, meine 
Füße, wird gefragt, welcher Körperteil bei bestimmten, benannten Kindern noch müde aussieht und wachgeschüttelt werden müsste. Dafür werden zwei kontrastierende Optionen vorgegeben: Sein Bauch oder seine Zunge? Ihr Mund oder ihre Nase (die Kinder finden es durchaus belustigend, Wege zu finden, wie die Nase wachgeschüttelt werden kann). Die Förderkraft achtet beim Nachfragen darauf, dass sie alle vorgeschlagenen Optionen auch selbst zeigt und die Zielstruktur möglichst in hoher Frequenz wiederholt. Die Kinder können damit die geforderte Struktur sofort nutzen. Sie sehen zudem gleich das Resultat ihrer Entscheidung: Das Kind schüttelt den Körperteil wach, den die anderen bestimmt haben. Es schließt sich ein Raumlauf an, der thematisch schon auf die darauf folgende Weltreise einstimmt. Eine der Förderkräfte übernimmt dabei die Rolle der Kommentatorin, die andere animiert durch ihr körperliches und verbales Mitmachen. Sie führt mit den Kindern aus, was die Kommentatorin vorgibt:

Toni, Damla und Arif sind am Strand und spazieren. Die Sonne scheint auf Arifs Haut. Seine Haut darf nicht verbrennen. Er braucht seinen Sonnenhut. Autsch! Der Sand ist so heiß! Damla muss ganz schnell laufen. Ihre Füße tun weh!

In der folgenden Motivationsphase wird gemeinsam ein Globus betrachtet und nach Lieblingsländern gefragt. Dabei raten die Kinder: Guckt euch mal Melih an: Was denkt ihr, was sein Lieblingsland ist? Ist sein Lieblingsland Thailand oder ist sein Lieblingsland Marokko? Die Kinder antworten darauf. Hierfür liegen vor dem Globus schon Kommunikationskarten mit der gewünschten Struktur aus. Mit ihren eigenen Äußerungen sichern die Förderkräfte ab, dass klimatisch unterschiedliche Länder genannt werden. Sollten nur warme Länder gewählt werden, bezeichnen die Förderkräfte besonders regnerische oder verschneite Länder als ihre Lieblingsländer. Danach werden die SchülerInnen befragt, ob sie wissen, wie das Wetter in ihrem Lieblingsland meistens sei, wobei die Förderkräfte immer wieder nachhaken: Aha, es ist immer warm. Ist es denn dort auch im Winter warm oder schneit es dann? Was denkst du? Anschließend sollen alle beantworten, ob sie die genannten Länder schon bereist haben. Sobald eines der Kinder oder eine der Förderkräfte diese Frage verneint, kann die andere Förderkraft enthusiastisch den Vorschlag einbringen, gemeinsam eine Reise in das Land zu unternehmen und dort zu entdecken, wie das Wetter ist. Im Gespräch mit den Kindern kann weiterhin entschieden werden, dass es am spannendsten ist, eine Weltreise vorzunehmen und verschiedene Länder mit unterschiedlichem Wetter kennenzulernen. Dabei wird zur Strukturvermittlungsphase übergeleitet. Denn im Austausch über die künftige Weltreise, können die Förderkräfte den Kindern die Wichtigkeit vermitteln, die einer genauen Planung bei einer langen Reise gebührt. Dazu gehört auch, zu überlegen, was sich im Gepäck befinden muss. Hierfür wird gemeinsam pantomimisch ein Koffer gepackt. Jeder/jede zeigt, was er/sie braucht, wenn Länder bereist werden, die ganz unterschiedliches Wetter haben: Regen, Sonne, Eis und Schnee. Für das pantomimische Präsentieren 
der Gegenstände liegt in der Mitte des Raums der große (erdachte) Koffer. Direkt nach dem Verstauen der Utensilien im Koffer gibt es für jeden/jede einen kurzen Applaus. Anschließend wird in die Runde gefragt: Was ist jetzt von $X$ im Koffer?, um gleich darauf eine Alternativfrage folgen zu lassen wie beispielsweise: Seine Sonnencreme oder sein Sonnenhut? Die Alternativfrage ist eine aus der Sprachtherapie bekannte Modellierungstechnik. Bei richtigem Einsatz kann sie kognitiv entlasten, so dass sich die Kinder auf die inhaltliche Entscheidung konzentrieren können. Gleichzeitig ist sichergestellt, dass die Kinder die Zielstruktur unmittelbar vor der eigenen Antwort korrekt hören, was eine fehlerfreie Reproduktion wahrscheinlicher macht. Durch den korrekten Output wird wiederum das Verinnerlichen der neuen Struktur begünstigt. Die Kinder wählen also in der Übung zum Kofferinhalt aus zwei angebotenen Möglichkeiten die ihnen richtig erscheinende Antwort aus und wiederholen diese laut. Im anschließenden Reiseplanungskomitee wird gemeinsam mithilfe einer großen Checkliste (Plakat) schriftlich festgehalten, was sich im Gepäck befindet. Nach der Frage Was ist von $X$ im Koffer? und den gesammelten Antworten wird explizit darauf eingegangen, wo die strukturellen Unterschiede liegen. Wann sagt man sein, wann ihr? Wann seine und wann ihre? Abbildung 5 zeigt eine mögliche Checkliste.

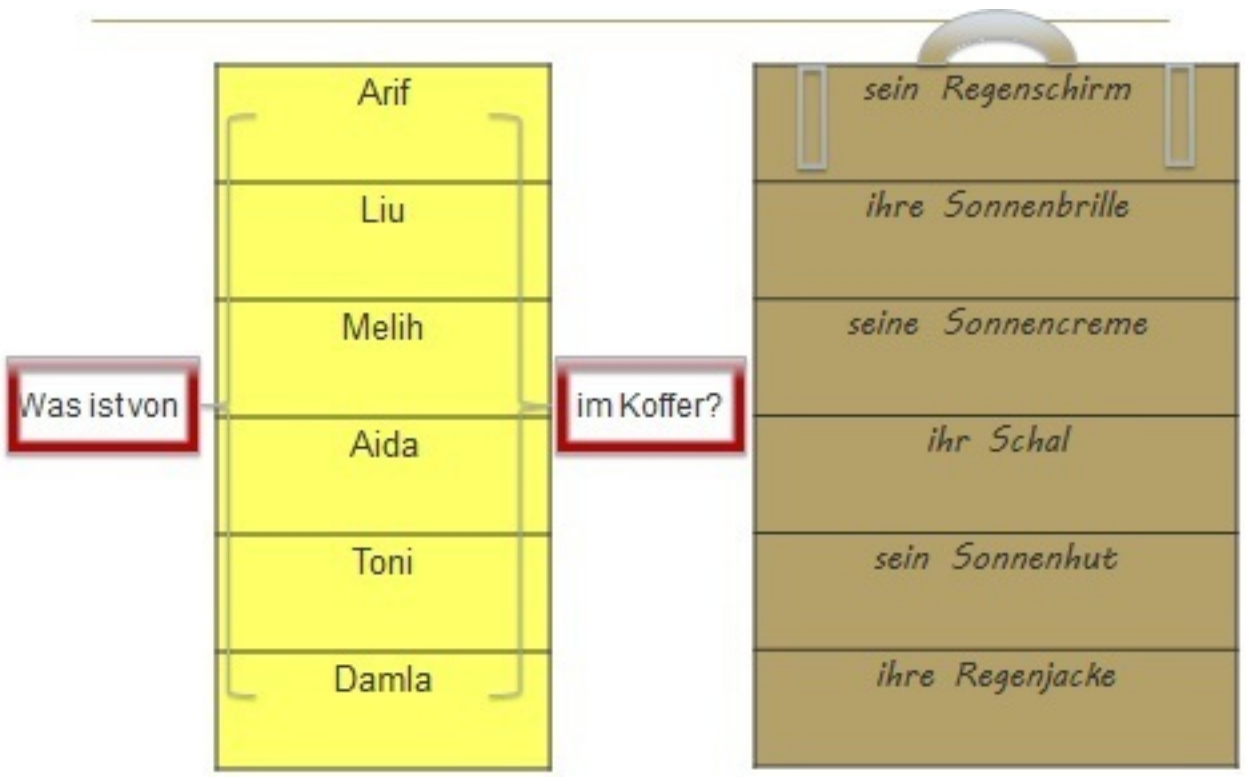

Abbildung 5: Checkliste - was ist im Koffer?

Ist die Arbeit an der Checkliste beendet, folgt die Strukturanwendung. Hierfür begeben sich alle auf eine improvisierte Weltreise. Während der ganzen Zeit fungiert eine Förderkraft moderierend als Reiseführerin, die gewisse Szenarien vorgibt. Hierauf müssen die Kinder reagieren. So kann beispielsweise im ersten Land ein Gewitter aufziehen. Die Förderkraft fordert Sprachproduktionen der Kinder im Spiel durch Ausrufe wie Schaut mal - da hinten kommen dunkle 
Wolken. Damla hat vorhin etwas in den Koffer gelegt. Das brauchen wir jetzt. Was war das? Zur Hilfe kann die zweite Förderkraft auf die Liste verweisen und die Kinder beim Antworten unterstützen (z.B. ins Ohr flüstern). Gemeinschaftlich werden die Gegenstände im erdachten Koffer nacheinander genutzt und im Spiel vervielfältigt, damit jedes Kind ein Objekt zur Verfügung hat. Innerhalb der Improvisation sollen die TeilnehmerInnen zusätzlich viele pantomimische Fotos von ihren Lieblingsorten schießen. Eine Förderkraft hebt dann während des Spiels ein Erlebnis als besonders dramatisch hervor. Zum Beispiel kann auf einer Bootsfahrt der Koffer über Bord gehen. Infolgedessen müssen alle Dinge aus dem Wasser gefischt und erneut zugeordnet werden. Mit Zeigegesten auf zwei Kinder kann eine Förderkraft hier fragen: War das sein Schirm oder ihr Schirm? Die Strukturvorgabe ist hier so eng, dass die Kinder Antworten in der gewünschten Form produzieren müssen. Am Ende dieser vierten Phase werden die unterschiedlichen possessiven Artikelwörter noch einmal notiert, indem alle Kinder in einem speziellen Lückentext die dramatischen Erlebnisse in Form eines Tagebucheintrags verarbeiten und von den verlorengegangenen Gegenständen berichten.

In der Präsentationsphase schauen sich die Kinder und die Förderkräfte das Fotoalbum der Reise an. Die TeilnehmerInnen präsentieren in Standbildern die Höhepunkte ihrer Unternehmungen. Kommentiert werden die „Fotos“ gemeinsam. Eine Förderkraft moderiert und stellt Fragen: Hier sehen wir Liu. Was ist gerade passiert? Warum weint sie? Die Antworten der Kinder fasst sie zusammen und wiederholt sie bestätigend in korrekter Form: Ja, das stimmt! Sie weint, weil ihr Regenschirm gerade ins Wasser gefallen ist. Jedes Foto wird mit einem Applaus honoriert. Die kommenden Fördereinheiten sollen zunehmend mehr Gelegenheiten für den freien Gebrauch der Zielstrukturen bieten.

In der abschließenden Reflexion wird ein Erzählstein herumgereicht und jedes Kind hat die Gelegenheit mitzuteilen, wie es ihm jetzt geht, was ihm an der Theaterstunde gefallen hat und was nicht. Dadurch findet es gleichzeitig den Übergang aus der Theaterwelt hinein in die Alltagswelt.

\section{3 Überprüfung der Wirksamkeit}

Um Aussagen über die Wirksamkeit der Maßnahme treffen zu können, müssen die Daten der Interventionsgruppe mit denen einer Kontrollgruppe verglichen werden. In der Kontrollgruppe sind Kinder aus dem gleichen schulischen Kontext mit vergleichbaren sprachlichen Fähigkeiten, die allerdings nicht an dieser Fördermaßnahme teilnehmen. Bei der vorgestellten dramagrammatischen Intervention handelt sich um ein Angebot fachsensibler Sprachförderung, das die AG-TeilnehmerInnen auf die (fach-)sprachlichen Anforderungen der Sekundarstufe I vorzubereiten versucht. Die sprachliche Entwicklung wird immer auch in Verbindung mit fachlichen Inhalten gefördert. Zu erwarten wäre daher, dass die Kinder der Interventionsgruppe sowohl in der Sprachkompetenz als auch in der Fachkompetenz gegenüber den Kindern der Kontrollgruppe einen Vorsprung erzielen. Für beide Bereiche sind verschiedene Messungen 
vorgesehen, die in nachstehender Tabelle zusammengefasst sind. In Klammern sind die Testzeitpunkte bzw. Überprüfungsintervalle angegeben.

Wir hoffen, durch diesen sehr umfassenden Testkatalog die Wirksamkeit der dramagrammatischen Methode in den verschiedensten Ausprägungen erfassen und dokumentieren zu können, um (im Falle positiver Evaluationsergebnisse) gegenüber der Stadt Tübingen für die Fortsetzung des hier präsentierten Nachhaltigkeitskonzepts argumentieren zu können.

Tabelle 2: Verfahren zur Überprüfung sprachlicher und fachlicher Kompetenzen

\begin{tabular}{ll}
\hline Sprachliche Kompetenz & Sachkompetenz im Fach MeNuK \\
\hline $\begin{array}{l}\text { Standardisierte Tests für die Bereiche } \\
\begin{array}{l}\text { Grammatik, Leseverständnis, Textproduktion } \\
\text { (am Anfang und am Ende der Intervention) }\end{array}\end{array}$ & $\begin{array}{l}\text { in Absprache mit den Lehrern } \\
\text { Klassenarbeiten im Fach } \\
\text { (halbjährlich) }\end{array}$ \\
$\begin{array}{l}\text { Zusatzdiagnostiken zu einzelnen } \\
\begin{array}{l}\text { Förderschwerpunkten } \\
\text { (in regelmäßigen Abständen) }\end{array}\end{array}$ & $\begin{array}{l}\text { Aufgaben zu Themen aus der AG } \\
\text { unter Einbeziehung behandelter Operatoren } \\
\text { (in regelmäßigen Abständen) }\end{array}$ \\
$\begin{array}{l}\text { Lehrerfragebogen zur Einschätzung } \\
\text { mündlicher und schriftlicher }\end{array}$ & $\begin{array}{l}\text { Lehrerfragebogen zur Einschätzung des } \\
\text { fachlichen Wissens und der Mitarbeit im }\end{array}$ \\
$\begin{array}{l}\text { Sprachfähigkeiten } \\
\text { (jährlich) }\end{array}$ & $\begin{array}{l}\text { Fachunterricht } \\
\text { (jährlich) }\end{array}$ \\
$\begin{array}{l}\text { Schülerfragebogen zur Selbsteinschätzung } \\
\text { mündlicher und schriftlicher }\end{array}$ & $\begin{array}{l}\text { Schülerfragebogen zur Lernfreude im Fach, } \\
\text { zur Selbsteinschätzung des fachlichen } \\
\text { Sprachfähigkeiten } \\
\text { (jährlich) }\end{array}$ \\
\hline
\end{tabular}

\section{Bibliografie}

Arbeitsgruppe Bildungsberichterstattung (2012): Bildung in Deutschland 2012. Bielefeld: Bertelsmann

Batzel, Andrea; Bohl, Torsten \& Bryant, Doreen (2013): Evaluation des Tübinger Theatercamps „Stadt der Kinder“. Ein Ferienprojekt zur Förderung von Sprache und sozialer Kompetenz. Hohengehren: Schneider Verlag

Becker-Mrotzek, Michael; Hentschel, Britta; Hippmann, Kathrin \& Linnemann, Markus (2012): Sprachförderung in deutschen Schulen - die Sicht der Lehrerinnen und Lehrer. Ergebnisse einer Umfrage durchgeführt von IPSOS (Hamburg)

[http://www.mercator-institut-sprachfoerderung.de/fileadmin/ user_upload/Lehrerumfrage_Langfassung_final_30_05_03.pdf, letzter Zugriff 10.10.2015] 
Becker-Mrotzek, Michael; Schramm, Karen; Thürmann, Eike \& Vollmer, Helmut Johannes (Hrsg.) (2013): Sprache im Fach. Sprachlichkeit und fachliches Lernen. Münster: Waxmann

Benholz, Claudia; Kniffka, Gabriele \& Winters-Ohle, Elmar (Hrsg.) (2010): Fachliche und sprachliche Förderung von Schülern mit Migrationsgeschichte. Münster: Waxmann

Benholz, Claudia \& Rau, Sarah (2011): Möglichkeiten der Sprachförderung im Sachunterricht [https://www. uni-due.de/imperia/md/content/prodaz/ sprachfoerderung_sachunterricht_grundschule.pdf, letzter Zugriff 10.10.2015]

Bolton, Gavin M. (1984): Drama as Education. An argument for placing drama at the centre of the curriculum. Harlow: Longman

Bonnet, Andreas \& Küppers, Almut (2011): Wozu taugen kooperatives Lernen und Dramapädagogik? Vergleich zweier populärer Inszenierungsformen. In: Küppers, Almut; Schmidt, Torben \& Walter, Maik (Hrsg.): Inszenierungstechniken im Fremdsprachenunterricht. Grundlagen, Formen, Perspektiven. Braunschweig: Bildungshaus Schulbuchverlage, 32-5

Bryant, Doreen (2015): Deutsche Relativsatzstrukturen als Lern- und Lehrgegenstand. In: Wöllstein, Angelika (Hrsg.): Das Topologische Modell für die Schule. Baltmannsweiler: Schneider Verlag Hohengehren, 77-99

Bryant, Doreen (2013): Das Tübinger Theatercamp als Lehr-, Lern- und Forschungsplattform. In: Zeitschrift für Theaterpädagogik 63, 36-37

Bryant, Doreen (2012): DaZ und Theater. Der dramapädagogische Ansatz zur Förderung der Bildungssprache. In: Scenario - Zeitschrift für Drama- und Theaterpädagogik in der Fremd- und Zweitsprachenvermittlung 1, 27-55

Bürkert, Joachim (2011): Theater im DaF-Unterricht. In: Miteinander. Informationen des Verbandes der Deutsch-Lehrenden Litauens 2, 13-17

Dauviller, Christa \& Lévy-Hillerich, Dorothea (2007): Spiele im Deutschunterricht. 3. Aufl. Berlin / München: Langenscheidt

DICE Consortium (Hrsg.) (2010): The DICE has been cast. Research findings and recommendations on educational theatre and drama. Budapest: European Commission [www . dramanetwork . eu, letzter Zugriff 30.08.2013]

Erman, Britt \& Warren, Beatrice (2000): The idiom principle and the open choice principle. Text 20, 501-524

Even, Susanne (2003): Drama Grammatik. Dramapädagogische Ansätze für den Grammatikunterricht Deutsch als Fremdsprache. München: Iudicium

Expertenrat „Herkunft und Bildungserfolg“ [Baumert, Jürgen et al.] (2011): Empfehlungen für Bildungspolitische Weichenstellungen in der Perspektive auf das Jahr 2020. Stuttgart: Ministerium für Kultus Jugend und Sport Baden-Württemberg

Feilke, Helmuth (2012): Bildungssprachliche Kompetenzen - fördern und entwickeln. Praxis Deutsch 233, 4-13 
Gibbons, Pauline (2002): Scaffolding Language, Scaffolding Learning. Teaching Second Language Learners in the Mainstream Classroom. Portsmouth, NH: Heinemann

Gogolin, Ingrid; Dirim, Inci; Lange, Imke; Lengyel, Drorit; Michel, Ute; Neumann, Ursula; Reich, Hans H.; Roth, Hans-Joachim \& Schwippert, Knut (2011): Förderung von Kindern und Jugendlichen mit Migrationshintergrund FörMig. Bilanz und Perspektiven eines Modellprogramms. Münster:

Waxmann

Handwerker, Brigitte \& Madlener, Karin (2009): Chunks für DaF. Theoretischer Hintergrund und Prototyp einer multimedialen Lernumgebung.

Hohengehren: Schneider Verlag

Heathcote, Dorothy \& Bolton, Gavin (1994): Drama for Learning: Dorothy Heathcote's Mantle of the Expert Approach to Education. Dimensions of Drama Series. Portsmouth: Heinemann

Kanngießer-Krebs, Gisela (2013): Szenisches Spiel. In: Der Fremdsprachliche Unterricht Spanisch 42, 4-11

Kinze, Julia (2012): Das HamburgerTheaterSprachCamp - Methoden und Ergebnisse der Evaluation. In: Scenario 2012-1, 85-102

[http://publish.ucc.ie/scenario/2012/01/kinze/06/de, letzter Zugriff 10.10.2015]

Küppers, Almut \& Walter, Maik (2012): Theatermethoden auf dem Prüfstand der Forschung. In: Scenario 2012-1, 1-9

Landeselternbeirat Baden-Württemberg (2014): Stellungnahme [http://leb-bw.de/infosdownloads/cat_view/1-stellungnahmen/47stellungnahmen-2014?limit $=15 \&$ order $=$ name\&dir $=$ ASC\&start $=10$, letzter Zugriff 21.10.2015]

Landesakademie für Fortbildung und Personalentwicklung an Schulen Baden-Württemberg: Basisoperatorenkatalog in den gesellschaftswissenschaftlichen Fächern in Baden-Württemberg [http: //lehrerfortbildung-bw.de/faecher/gwg/fbl/modull/geo/operator/, letzter Zugriff 22.10.2015]

Landesinstitut für Schulentwicklung (2011): Abschlussbericht Evaluation der Fächerverbünde [http://www.1s-bw.de/Handreichungen/pub_online/ Bericht_Evaluation_Eaecherverbuende.pdf, letzter Zugriff 21.10.2015]

Lechthaler, Katja (2004): Alle Kinder spielen gern Theater. Was Kinder beim Schauspielern erleben und lernen. Wiesbaden: Marixverlag

Ministerium für Kultus, Jugend und Sport Baden-Württemberg (2004): Bildungsplan Grundschule [http://www.bildung-staerkt-menschen.de/service/downloads/ Bildungsplaene/Grundschule/Grundschule_Bildungsplan_Gesamt.pdf, letzter Zugriff 21.10.2015]

Neumann, Ursula; Priebe, Birte; Ruf, Irinell; Kinze, Julia \& Dluhosch, Carina (2012): TheaterSprachKurse Hamburg im Anschluss an das Hamburger 
TheaterSprachCamp 2012 [https://www.ew.uni-hamburg.de/ueber-diefakultaet/personen/neumann/files/tsk-konzept-final.pdf, letzter Zugriff 15.10.2015]

Pawley, Andrew \& Syder, Frances (1983): Two puzzles for linguistic theory: Nativlike selection and nativelike fluency. In: Richards, Jack C. \& Schmidt, Richard W. (Hrsg.): Language and Communication. London and New York: Longman, 191-226

Quehl, Thomas \& Trapp, Ute (2013): Sprachbildung im Sachunterricht der Grundschule. Mit dem Scaffolding-Konzept unterwegs zur Bildungssprache. Münster: Waxmann

Ricart Brede, Julia (2015): Zur Didaktik des Versuchsprotokolls als Aufgabe eines sprachsensiblen Fachunterrichts und eines fachsensiblen Sprach(förder)unterrichts. In: Klages, Hana \& Pagonis, Giulio (Hrsg.): Linguistisch fundierte Sprachförderung und Sprachdidaktik. Grundlagen, Konzepte, Desiderate. Berlin / München / Boston: De Gruyter, 173-190

Ritterfeld, Ute (2000): Welchen und wieviel Input braucht das Kind? In: Grimm, Hannelore (Hrsg.): Sprachentwicklung. Enzyklopädie der Psychologie (Bd. C3/3). Göttingen: Hogrefe Verlag, 403-432

Sambanis, Michaela (2011): Weniger stillsitzen, mehr lernen? - Effekte bewegungsbasierter Wortschatzarbeit auf der Primar- und Sekundarstufe. In: Schäfer, Patrick \& Schowalter, Christine (Hrsg.): In mediam linguam. Mediensprache - Redewendungen - Sprachvermittlung. Festschrift für Heinz-Helmut Lüger. Landau: Verlag Empirische Pädagogik, 365-376

Schewe, Manfred (1993): Fremdsprache inszenieren: Zur Fundierung einer dramapädagogischen Lehr- und Lernpraxis. Zentrum für pädagogische Berufspraxis. Universität Oldenburg: BIS-Vlg

Slobin, Dan I. (1973): Cognitive prerequisites for the development of grammar. In: Ferguson, Charles A. \& Slobin, Dan I. (Hrsg.): Studies of child language development. New York: Holt, Rinehart \& Winston, 175-208

Stengers, Helene; Boers, Frank; Housen, Alex \& Eyckmans, June (2011): Formulaic sequences and L2 oral proficieny. Does the type of target language influence the association? In: IRAL 49, 321-343

Sting, Wolfgang (2012): Performance und Theater als anderes Sprechen. In: Scenario 2012 Heft 1, 54-63 [http://publish.ucc.ie/scenario/2012/01/sting/04/de, letzter Zugriff 10.10.2015] 